\title{
Influence of the controllability of electric vehicles on generation and storage capacity expansion decisions
}

\author{
Miguel Carrión ${ }^{\mathrm{a}, *}$, Ruth Domínguez ${ }^{\mathrm{a}}$, Rafael Zárate-Miñano ${ }^{\mathrm{b}}$ \\ ${ }^{a}$ Department of Electrical Engineering, Universidad de Castilla - La Mancha, Toledo, Spain \\ ${ }^{b}$ Department of Electrical Engineering, Universidad de Castilla - La Mancha, Almadén, Spain
}

\begin{abstract}
This paper proposes a capacity investment model to analyze the influence of the controllability of the charge of plug-in electric vehicles (PEVs) in generation and storage expansion decisions. The proposed model provides the financial incentives that should be offered to PEV users in order to implement the optimal expansion decisions. Considering that the decision-making process faced by the power system planner must simultaneously consider long- and short-term uncertainties, a three-stage stochastic programming problem is formulated. In this model, capacity investments and financial incentives for PEV users are decided in the first stage, whereas operating decisions regarding the day-ahead and real-time markets are made in the second and third stages, respectively. Numerical results are provided from a realistic case study based on the isolated power system comprising Lanzarote and Fuerteventura islands in Spain.
\end{abstract}

Keywords:

controlled charge, financial incentives, energy storage systems, long- and short-term uncertainties, stochastic programming

\section{Introduction}

The Intergovernmental Panel on Climate Change (IPCC) has proven the human responsibility for the ongoing global warming and claims radical changes

\footnotetext{
${ }^{*}$ Corresponding author. Tel.: +34925268800 (5750)

Email addresses: Miguel.Carrion@uclm.es (Miguel Carrión), Ruth.Dominguez@uclm.es (Ruth Domínguez), Rafael.Zarate@uclm.es (Rafael Zárate-Miñano)
} 
in the economic development [1]. Since the electricity generation is currently the cause of $30 \%$ of the global greenhouse gas emissions, mandatory changes are needed in this sector. For this reason, power systems have started a major transformation that will require a higher interaction between consumers and electricity suppliers in order to accommodate the expected massive electricity production from renewable energies.

The variability of the output of intermittent renewable sources, such as wind and solar radiation, may be compensated by an increase in the flexibility in the demand side. In this sense, the direct control of the charging processes of the batteries of plug-in electric vehicles (PEVs) may represent a useful instrument used by power system operators (SOs) to facilitate the operation of future power systems. However, the high acquisition cost of PEVs and the still poor development of charging infrastructures are currently the main barriers for spreading the use of PEVs. Considering the above, the development of capacity expansion models specially tailored for power systems with high presence of renewable generating units and controllable loads are of utmost importance.

The capacity expansion problem considering renewable units has been widely studied. For instance, [2] presents a novel stochastic programming problem to decide how a thermal-dominated power system can be transformed into a renewabledominated one. The impact of forecast errors of wind power production on the capacity expansion planning has been investigated in [3]. A classical two-stage stochastic programming is presented in [4] to analyze the effect of the uncertainty in capacity expansion decisions. Reference [5] proposes a multistage model to decide generation, storage and transmission investments considering simultaneously large- and small-scale uncertainties. A sample average approximation method for the capacity expansion problem is proposed in [6]. In [7], a generation and storage capacity expansion model that considers power ramps, minimum power output and minimum up and down times is presented. The authors of [8] propose a mixed-integer linear formulation for modeling a generation and transmission ex- 
pansion problem considering different time scales.

Some works have also analyzed the capacity expansion problem considering the presence of a large number of electric vehicles. For instance, reference [12] proposes a deterministic capacity expansion problem considering smart charge and discharge of electric vehicles. In [13], the capacity expansion problem for distribution systems considering storages and electric vehicle charging stations has been studied.

In this paper we assume the role of a power system planner that seeks to determine the optimal generation and storage investment decisions to be implemented on an existing power system. For doing that, a novel formulation for the generation and storage capacity problem considering a large presence of electric vehicles is proposed. In order to increase the flexibility in the operation of power systems, we assume that the system operator can exercise a direct control over the charging processes of PEVs in exchange for a financial reimbursement paid to PEV users. Considering this, the expansion plans determined by the power system planner are usually used to promote the installation of some technologies over others in a non-arbitrary manner. In this manner, the expansion plans decided by the system planner are useful either to design incentive policies or to determine quantitatively the maximum capacities of generation and storage technologies that are allowed to be installed by private investors in a given power system. Accordingly, the final decision about whether or not to invest in a given technology is only made by investors, not by system planners.

Since renewable generating units are becoming economically competitive, a capacity investment model especially tailored for power systems with a high presence of such technologies is required. This consideration is relevant because the availability of the power output of most frequent renewable technologies is variable, volatile and uncertain. Note that some recent works, as [3], have proven that modeling short-term uncertainties in capacity expansion problems may affect the investment decisions obtained for power systems with high presence of units 
with uncertain power outputs. For this reason, the capacity expansion model proposed in this paper considers the effect of the variability and uncertainty of the production of renewable units in the day-ahead energy and reserve capacity markets, as well as in the real-time energy market.

In order to increase the flexibility in the operation of power systems, we consider that SOs may exercise a direct control over the charging process of PEVs in exchange for a financial reimbursement that is paid to PEV users. The capabilities of PEVs to contribute in the integration of renewable sources have been investigated in [14]. The resulting number of controlled PEVs is a function of the total value of the offered incentives, whose optimal values are an output of the proposed model. These financial incentives represent additional costs for the power system operation that must be taken into account in the capacity expansion problem. Note that the effect of the presence of electric vehicles in the investments in transmission and distribution networks is not analyzed in this study. The control over the charging processes of PEVs could be implemented in practice through financial intermediaries as aggregators or retailers, [15]-[17]. Note that none of the works consulted proposes procedures to determine such incentives.

Finally, long-term uncertainties as the annual demand growth, capital costs of generating and storage units, the number of PEVs and the degree of willingness of PEV users to delegate the charge of their PEVs to the SO are explicitly characterized in this capacity expansion problem.

The structure of this paper is as follows. Section 2 describes the decision framework and the formulation of the proposed capacity expansion problem. Section 3 explains the solution procedure used to solve the resulting problem. In Section 4 numerical results are provided and discussed. Finally, in Section 5 the conclusions of this work are presented. 


\section{Mathematical formulation}

In this section we describe the generation and storage capacity expansion problem that is proposed to analyze the influence of controlling the charge of electric vehicles in the generation and storage capacity decisions.

\subsection{Initial assumptions}

The mathematical formulation described in this section is based on the following assumptions:

- Thermal and renewable power units and energy storage facilities are used to provide the energy demanded by traditional loads and electric vehicles.

- Renewable units are non dispatchable and provide intermittent power outputs.

- Generation offer and consumption bid curves are linear.

- The ability of loads to respond to pricing is very small and is therefore ignored.

- The transmission network is represented by the DC approximation [18].

- PEVs charge their batteries from the grid.

- PEV users are willing to leave the control of the charge and discharge of their vehicles if a large enough economic reimbursement is offered to them by the SO.

- The number of controlled vehicles only depends on the value of the offered incentive.

- The energy stored in the PEVs at the end of the charging period has to be greater than or equal to a minimum level defined by PEV users. 
- There exist appropriate communication facilities allowing the effective control of PEVs.

- The energy charged by those PEVs that are not controlled by the SO is modeled as an inelastic demand.

\subsection{Notation}

The notation used throughout the paper is included below for quick reference.

Sets and Indices

$C$ Sets of controlling modes of electric vehicles, indexed by $c$

$E$ Set of characteristic days, indexed by $e$

$I$ Set of generating and storage units, indexed by $i$

$I_{n}$ Set of generating and storage units located in bus $n$

$I^{\mathrm{G} / \mathrm{S}}$ Set of generating/storage units

$I^{\mathrm{G}, \mathrm{D} / \mathrm{I}}$ Set of dispatchable/intermittent generating units

$I_{n}^{\mathrm{G}, \mathrm{D} / \mathrm{I}}$ Set of dispatchable/intermittent generating units located in bus $n$

$I_{n}^{\mathrm{S}}$ Set of storage units located in bus $n$

$K$ Set of plug-in electric vehicle groups, indexed by $k$

$K_{t}$ Set of plug-in electric vehicle groups that are connected to the grid in period $t$

$L$ Set of transmission lines, indexed by $\ell$

$M$ Set of blocks in piecewise price-cuota curves, indexed by $m$

$N$ Set of buses, indexed by $n$

$T$ Set of time periods, indexed by $t$

$T_{k}$ Set of time periods in which PEVs group $k$ can be charged from or discharged to the grid 
$\nu$ Index used the denote the iteration counter in the Benders' decomposition algorithm

$\Xi$ Set of scenarios modeling long-term uncertainty, indexed by $\xi$

$\Omega$ Set of scenarios modeling short-term uncertainty, indexed by $\omega$

\section{Parameters}

$C_{\text {iet }}^{\mathrm{CU} / \mathrm{CD}}$ Offering cost of up/down reserve capacity of unit $i$ in characteristic day $e$, period $t$ and long-term scenario $\xi$

$C_{i e t \xi}^{\mathrm{D} / \mathrm{C}}$ Offering cost/consumption bid in the day-ahead energy market of unit $i$ in characteristic day $e$, period $t$ and long-term scenario $\xi$

$C_{i e t \xi}^{\mathrm{DU} / \mathrm{DD}}$ Offering cost of deploying up/down reserve of unit $i$ in characteristic day $e$, period $t$ and long-term scenario $\xi$

$C_{i \xi}^{\mathrm{G}, \mathrm{C}}$ Annualized capital cost of generating unit $i$ and long-term scenario $\xi$

$C_{i \xi}^{\mathrm{SE}, \mathrm{C}}$ Annualized capital cost of the energy component of storage unit $i$ in longterm scenario $\xi$

$C_{i \xi}^{\mathrm{SP}, \mathrm{C}}$ Annualized capital cost of the power component of storage unit $i$ in longterm scenario $\xi$

$C^{\mathrm{UD}}$ Cost of unserved demand

$L_{n e t \xi}^{\mathrm{D}}$ Demand in the day-ahead market in bus $n$, characteristic day $e$, period $t$ and long-term scenario $\xi$

$L_{k n e t \xi}^{\mathrm{EV}, \mathrm{D}}$ Demand in the day-ahead market of non-controlled electric vehicle group $k$ in bus $n$, characteristic day $e$, period $t$ and long-term scenario $\xi$

$L_{k n e t \xi \omega}^{\mathrm{EV}, \mathrm{R}}$ Demand in the real-time market of non-controlled electric vehicle group $k$ in bus $n$, characteristic day $e$, period $t$, long-term scenario $\xi$ and short-term scenario $\omega$

$L_{n e t \xi \omega}^{\mathrm{R}}$ Demand in the real-time market in bus $n$, characteristic day $e$, period $t$, long-term scenario $\xi$ and short-term scenario $\omega$ 
$N_{k n \xi}^{\mathrm{EV}}$ Number of electric vehicles pertaining to group $k$ and bus $n$ in long-term scenario $\xi$

$P_{\max }^{\mathrm{BPT}}$ Maximum charged/discharged power in the battery of a PEV $k$

$P_{k n e \xi \omega}^{\mathrm{BS}, 0 / \mathrm{F}}$ Initial/final status of the battery of PEV group $k$, at bus $n$, in characteristic day $e$, period $t$, long-term scenario $\xi$ and short-term scenario $\omega$

$P_{\max , k}^{\mathrm{EV}, \mathrm{S}}$ Maximum energy level stored in PEV group $k$

$S P_{\xi e}^{(\nu)}$ Parameter that is equal to the optimal objective function of the subproblem associated with long-term scenario $\xi$ and characteristic day $e$ in iteration $(\nu)$ of the Benders' decomposition algorithm

$P_{\text {min, }, k}^{\mathrm{EV}}$ Minimum energy level stored in PEV group $k$

$P_{\mathrm{up} / \mathrm{dw}, i}^{\mathrm{G}}$ Upper/down ramp factor of generating unit $i$

$P_{\max , \ell}^{\mathrm{L}}$ Capacity of transmission line $\ell$

$t_{k}^{\mathrm{I} / \mathrm{F}}$ Initial/final charging time of the battery of PEVs group $k$

$U_{\text {iet }}^{\mathrm{D}}$ Availability of intermittent unit $i$ in the day-ahead market in characteristic day $e$ and period $t$

$U_{\text {ietw }}^{\mathrm{R}}$ Availability of intermittent unit $i$ in characteristic day $e$, period $t$ and shortterm scenario $\omega$

$w_{d}$ Weight of characteristic day $d$

$X_{\ell}$ Reactance of line $\ell$

$\hat{X}_{k c m \xi}^{\mathrm{EV}}$ Auxiliary parameter used to compute the per unit number of PEVs in group $k$ pertaining to charging mode $c$, block $m$ and long-term scenario $\xi$

$z_{\text {lo }}^{(\nu)}$ Lower bound of problem (P1) computed in iteration $(\nu)$ of the Benders' decomposition algorithm

$z_{\text {up }}^{(\nu)}$ Upper bound of problem (P1) computed in iteration $(\nu)$ of the Benders' decomposition algorithm

$\gamma_{i}^{\mathrm{SS}, 0 / \mathrm{E}}$ Factor used to model the initial/ending status of the storage unit $i$ 
$\gamma_{i}^{\mathrm{S}, \mathrm{EP}}$ Relationship between energy and power capacities in storage unit $i$

$\gamma_{i}^{\text {SSmin }}$ Factor used to model the minimum energy that must contain storage unit $i$

$\eta^{\mathrm{EV}}$ Efficiency of charging/discharging PEVs

$\eta_{i}^{\mathrm{S}}$ Efficiency of charging/discharging storage unit $i$

$\hat{\lambda}_{k c m \xi}^{\mathrm{EV}}$ Auxiliary parameter used to compute the incentive offered to electric vehicle group $k$ in charging mode $c$, block $m$ and long-term scenario $\xi$

$\pi_{d \omega}$ Probability of short-term scenario $\omega$ in day $d$

$\tau_{\xi}$ Probability of long-term scenario $\xi$

\section{Variables}

$c_{i e t \xi}^{\mathrm{U} / \mathrm{D}} \mathrm{Up} /$ down reserve capacity scheduled by generating or storage unit $i$ in the day-ahead market, characteristic day $e$, period $t$ and long-term scenario $\xi$

$p_{\text {iet } \xi}^{\mathrm{C}}$ Consumption power scheduled by storage unit $i$ in the day-ahead market, in characteristic day $e$, period $t$ and long-term scenario $\xi$

$p_{\text {iet } \xi}^{\mathrm{D}}$ Generation power scheduled by unit $i$ in the day-ahead market, in characteristic day $e$, period $t$ and long-term scenario $\xi$

$p_{k n e t \xi \omega}^{\mathrm{EV}, \mathrm{D} / \mathrm{D}}$ Power charged/discharged from the batteries of PEV group $k$ in bus $n$, characteristic day $e$, period $t$, long-term scenario $\xi$ and short-term scenario $\omega$

$p_{k n e t \xi}^{\mathrm{EV}, \mathrm{DD} / \mathrm{DC}} \quad$ Power discharged/charged in the day-ahead market by PEV group $k$, in bus $n$, characteristic day $e$, period $t$ and long-term scenario $\xi$

$p_{k n e t \xi \omega}^{\mathrm{EV}, \mathrm{S}}$ Energy level in the batteries of PEV group $k$ in bus $n$, characteristic day $e$, period $t$, long-term scenario $\xi$ and short-term scenario $\omega$

$p_{i}^{\mathrm{G}, \mathrm{C}}$ Capacity built of generating unit $i$

$p_{\ell e t \xi}^{\mathrm{L}, \mathrm{D}}$ Power flow through line $\ell$ in the day-ahead market, characteristic day $e$, period $t$ and long-term scenario $\xi$ 
$p_{\ell e t \xi \omega}^{\mathrm{L}, \mathrm{R}}$ Power flow through line $\ell$ in the day-ahead market, in characteristic day $e$, period $t$, long-term scenario $\xi$ and short-term scenario $\omega$

$p_{i e t \xi \omega}^{\mathrm{R}}$ Power generated in the real-time operation by generating unit $i$, in characteristic day $e$, period $t$, long-term scenario $\xi$ and short-term scenario $\omega$

$p_{i e t \xi \omega}^{\mathrm{SC} / \mathrm{SD}}$ Power charged/discharged by storage $i$ in characteristic day $e$, period $t$, long-term scenario $\xi$ and short-term scenario $\omega$

$p_{i}^{\mathrm{SE}, \mathrm{C}}$ Energy capacity built of storage unit $i$

$p_{i}^{\mathrm{SP}, \mathrm{C}}$ Peak power of storage unit $i$

$p_{\text {iet } \xi \omega}^{\mathrm{SS}}$ Energy level in storage $i$, day $e$, period $t$, long-term scenario $\xi$ and shortterm scenario $\omega$

$p_{n e t \xi \omega}^{\mathrm{UD}, \mathrm{R}}$ Unserved demand in bus $n$ in the real-time operation, in characteristic day $e$, period $t$, long-term scenario $\xi$ and short-term scenario $\omega$

$r_{k n e t \xi \omega}^{\mathrm{EV} / \mathrm{D}} \mathrm{Up} /$ down reserve deployed by PEV group $k$ in bus $n$, characteristic day $e$, period $t$, long-term scenario $\xi$ and short-term scenario $\omega$

$r_{k n e t \xi \omega}^{\mathrm{EV}, \mathrm{UC} / \mathrm{DC}} \mathrm{Up} /$ down reserve deployed by PEV group $k$ in bus $n$ from charging in characteristic day $e$, period $t$, long-term scenario $\xi$ and short-term scenario $\omega$

$r_{k n e t \xi \omega}^{\mathrm{EV}, \mathrm{UD} / \mathrm{DD}} \mathrm{Up} /$ down reserve deployed by PEV group $k$ in bus $n$ from the discharging, characteristic day $e$, period $t$, long-term scenario $\xi$ and short-term scenario $\omega$

$r_{i e t \xi \omega}^{\mathrm{U} / \mathrm{D}}$ Deployed up/down reserve in the real-time operation by generating or storage unit $i$, in characteristic day $e$, period $t$, long-term scenario $\xi$ and shortterm scenario $\omega$

$r_{i e t \xi \omega}^{\mathrm{UC} / \mathrm{DC}} \mathrm{Up} /$ down reserve deployed in the real-time operation from the charge of storage unit $i$, in characteristic day $e$, period $t$, long-term scenario $\xi$ and short-term scenario $\omega$ 
$r_{i e t \xi \omega}^{\mathrm{UD} / \mathrm{DD}} \mathrm{Up} /$ down reserve deployed in the real-time operation from the discharge of storage unit $i$, in characteristic day $e$, period $t$, long-term scenario $\xi$ and short-term scenario $\omega$

$s_{\text {iet } \xi}^{\mathrm{D}}$ Power spillage of intermittent generating unit $i$ in the day-ahead market, in characteristic day $e$, period $t$ and long-term scenario $\xi$

$s_{i e t \xi \omega}^{\mathrm{R}}$ Power spillage of intermittent generating unit $i$ in the real-time market, in characteristic day $e$, period $t$, long-term scenario $\xi$ and short-term scenario $\omega$

$v_{k c n m}^{\mathrm{EV}}$ Binary variable that is equal to 1 if the incentive offered to PEV group $k$, in charging mode $c$, in bus $n$ belongs to block $m$ of the price-quota curve, being 0 otherwise

$x_{k c n \xi}^{\mathrm{EV}, \mathrm{C}}$ Per-unit number of controlled PEVs in group $k$, charging mode $c$, bus $n$ and long-term scenario $\xi$

$\alpha_{k n \xi e}^{\mathrm{EV}, \mathrm{C}}$ Dual variable associated with constraint (71)

$\alpha_{i e \xi}^{\mathrm{G}, \mathrm{C}}$ Dual variable associated with constraint (68)

$\alpha_{i e \xi}^{\mathrm{SE}, \mathrm{C}}$ Dual variable associated with constraint (69)

$\alpha_{i e \xi}^{\mathrm{SP}, \mathrm{C}}$ Dual variable associated with constraint (70)

$\beta$ Auxiliary variable used to estimate the expected operation costs for given first-stage variables in the Benders' decomposition algorithm

$\lambda_{k n c}^{\mathrm{EV}}$ Incentive offered to electric vehicle group $k$ in bus $n$ and charging mode $c$ $\lambda_{\text {kncm }}^{\mathrm{EV}, \mathrm{A}}$ Auxiliary variable used to compute the incentive offered to electric vehicle group $k$ in bus $n$, charging mode $c$ and block $m$

$\theta_{\text {net } \xi}^{\mathrm{L}, \mathrm{D}}$ Voltage angle of bus $n$ in the day-ahead market, characteristic day $e$, period $t$ and long-term scenario $\xi$

$\theta_{n e t \xi \omega}^{\mathrm{L}, \mathrm{R}}$ Voltage angle of bus $n$ in the real-time market, in characteristic day $e$, period $t$, long-term scenario $\xi$ and short-term scenario $\omega$ 


\subsection{Decision framework and planning horizon}

We consider a power system planner that desires to determine the optimal generating mix to procure the demand in a specified target year at minimum cost. To achieve this objective, the planner has to decide the capacity to install from a set of thermal and renewable candidate generating units. Renewable units are assumed to be non dispatchable with intermittent power output. In order to facilitate the incorporation of a large number of renewable units, energy storage systems can be also installed to increase the flexibility of the power system operation. Note that energy storage systems are adequate for performing a temporal arbitrage of energy, as well as providing up and down reserves.

Together with storage units, an appropriate management of controllable loads can provide additional flexibility to the power system operation. In this sense, PEVs present great ability for providing flexibility since i) their consumption of energy from the grid does not occur when they are at usage, ii) they are parked the major part of the day, iii) the processes of charging and discharging their batteries can be automatically controlled, and iv) they have a relatively large capacity of storage. In this manner, we assume that the $\mathrm{SO}$ will be able to control the charging process of a set of PEVs in exchange for a financial reimbursement paid to PEV users. The willingness of PEV users to be controlled by the SO is dependent on the offered financial incentive. Additionally, PEVs are classified in different groups according to their usage pattern, so that all PEVs belonging to the same group are assumed to have similar charging and discharging profiles.

We assume that the power system operation is settled in two stages. The first-stage represents the day-ahead market in which the scheduling of energy and up and down reserve capacities for each market participant are assigned. The real-time market is characterized in the second-stage in which the deployment of the reserves is determined to counteract unexpected deviations of demand and intermittent power outputs.

Considering the above, the proposed generation and energy storage capacity 
expansion model aims at determining:

- The capacity to install for each candidate generating unit.

- The capacity to install for each candidate energy storage unit.

- The economic incentives to offer to those PEV users willing to delegate the control of the charge of their PEVs to the SO.

In this problem we distinguish between long- and short-term uncertainties. Long-term uncertain parameters are those concerning the whole planning horizon. For instance, the future values of demand growth, capital costs of generating and energy storage units and the number of PEVs are examples of long-term uncertain parameters. The hourly demand and the renewable output availability for the next day can be defined as short-term uncertain parameters. Long- and short-term uncertain parameters are characterized by two different sets of scenarios denoted by $\Xi$ and $\Omega$, respectively. Because of the very nature of long-term uncertain parameters, they can be statistically characterized by expert judgment using the available information provided in technical reports [19]. On the other hand, the scenarios used to characterize the short-term uncertain parameters can be generated using standard scenario generation techniques based on time series models [20].

Considering the above, the proposed investment model is formulated by the three-stage stochastic programming problem represented in Figure 1. As it can be observed in this figure, investment decisions on generating and storage units are made at the first stage. The storage capacity is defined in terms of power and energy. In this stage, the economic incentives offered to PEV users are also determined. The second and third stages are used to represent the daily power system operation. The second stage characterizes the day-ahead energy and reserve capacity markets where energy and reserve capacity are scheduled for each of the 24 hours of the next day. The third stage models the real-time market where the deployment of reserves are determined. As is customary in capacity 
expansion problems, the considered planning horizon is characterized by a set of characteristic days.

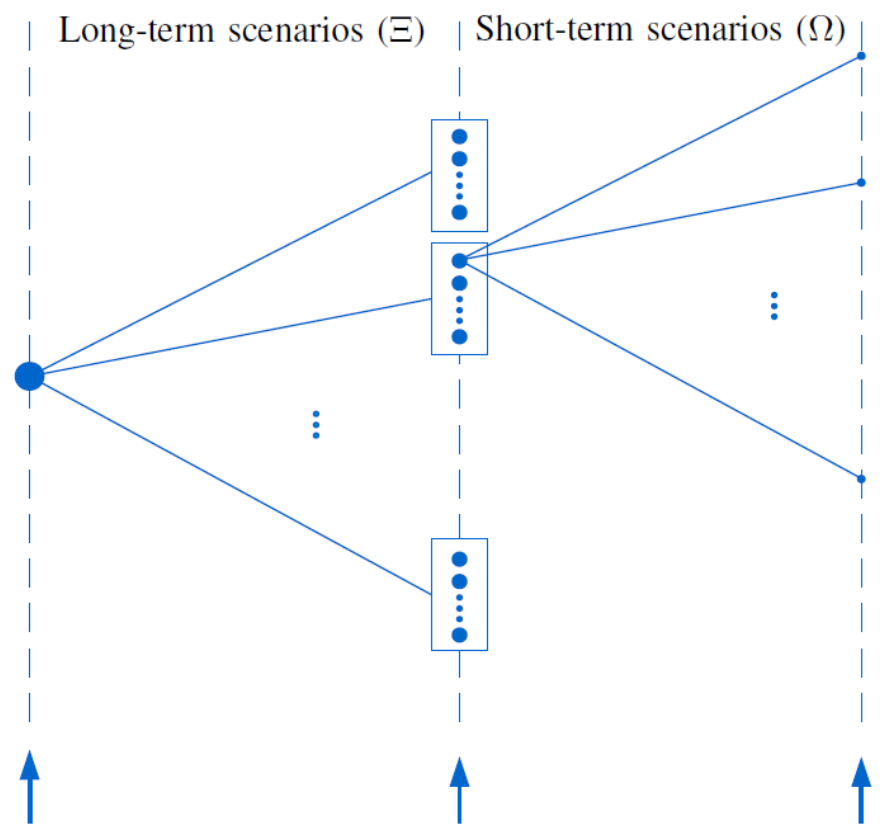

- Generating and storage

- Day-ahead market

- Real-time market investment decisions

- Financial incentive for PEVs

Figure 1: Decision-making process

\subsection{Three-stage stochastic programming formulation}

The mathematical formulation of the problem described above is the following:

$\operatorname{Minimize}_{\Theta}$

$$
\begin{aligned}
& \sum_{\xi \in \Xi} \tau_{\xi}\left[\sum_{i \in I^{\mathrm{G}}} C_{i \xi}^{\mathrm{G}, \mathrm{C}} p_{i}^{\mathrm{G}, \mathrm{C}}+\sum_{i \in I^{\mathrm{S}}}\left(C_{i \xi}^{\mathrm{SE}, \mathrm{C}} p_{i}^{\mathrm{SE}, \mathrm{C}}+C_{i \xi}^{\mathrm{SP}, \mathrm{C}} p_{i}^{\mathrm{SP}, \mathrm{C}}\right)+\right. \\
& \sum_{k \in K} \sum_{n \in N} \sum_{c \in C} \sum_{m \in M} N_{k n \xi}^{\mathrm{EV}} \hat{X}_{k m \xi}^{\mathrm{EV}} \lambda_{k c n m}^{\mathrm{EV}, \mathrm{A}}+ \\
& \sum_{e \in E} w_{e} \sum_{t \in T}\left(\sum_{i \in I^{\mathrm{G}, \mathrm{D} \cup I^{\mathrm{S}}}}\left(C_{i e t \xi}^{\mathrm{D}} p_{i e t \xi}^{\mathrm{D}}+C_{i e t \xi}^{\mathrm{CU}} c_{i e t \xi}^{\mathrm{U}}+C_{i e t \xi}^{\mathrm{CD}} c_{i e t \xi}^{\mathrm{D}}\right)+\right. \\
& \sum_{\omega \in \Omega} \pi_{e \omega}\left(\sum_{i \in\left\{I^{\left.\mathrm{G}, \mathrm{D} \cup I^{\mathrm{S}}\right\}}\right.}\left(C_{i e t \xi}^{\mathrm{DU}} r_{i e t \xi \omega}^{\mathrm{U}}-C_{i e t \xi}^{\mathrm{DD}} r_{i e t \xi \omega}^{\mathrm{D}}\right)+\right. \\
& \left.\left.\left.\sum_{n \in N} C^{\mathrm{UD}} p_{n e t \xi \omega}^{\mathrm{UD}}\right)\right)\right]
\end{aligned}
$$


Subject to:

- Investment constraints

$$
\begin{aligned}
& 0 \leq p_{i}^{\mathrm{G}, \mathrm{C}} \leq P_{\max , \mathrm{i}}^{\mathrm{G}, \mathrm{C}}, \quad \forall i \in I^{\mathrm{G}} \\
& 0 \leq p_{i}^{\mathrm{SE}, \mathrm{C}} \leq P_{\max , \mathrm{i}}^{\mathrm{SE}, \mathrm{C}}, \quad \forall i \in I^{\mathrm{S}} \\
& 0 \leq p_{i}^{\mathrm{SP}, \mathrm{C}} \leq P_{\max , \mathrm{i}}^{\mathrm{SP}, \mathrm{C}}, \quad \forall i \in I^{\mathrm{S}} \\
& p_{i}^{\mathrm{SE}, \mathrm{C}}=\gamma_{i}^{\mathrm{S}, \mathrm{EP}} p_{i}^{\mathrm{SP}, \mathrm{C}}, \quad \forall i \in I^{\mathrm{S}}
\end{aligned}
$$

- Financial incentive offered to PEVs

$$
\begin{aligned}
& \hat{\lambda}_{k c m-1}^{\mathrm{EV}} v_{k n c m}^{\mathrm{EV}} \leq \lambda_{k n c m}^{\mathrm{EV}, \mathrm{A}} \leq \hat{\lambda}_{k c m}^{\mathrm{EV}} v_{k n c m}^{\mathrm{EV}}, \forall k, \forall n, \forall c, \forall m \\
& \sum_{m \in M} v_{k n c m}^{\mathrm{EV}}=1, \quad \forall k, \forall n, \forall c \\
& \sum_{m \in M} \lambda_{k n c m}^{\mathrm{EV}, \mathrm{A}}=\lambda_{k n c}^{\mathrm{EV}}, \quad \forall k, \forall n, \forall c \\
& x_{k n c \xi}^{\mathrm{EV}, \mathrm{C}}=\sum_{m \in M} \hat{X}_{k m \xi}^{\mathrm{EV}} v_{k n c m}^{\mathrm{EV}}, \forall k, \forall n, \forall c, \forall \xi \\
& \sum_{c \in C} x_{k n c \xi}^{\mathrm{EV}, \mathrm{C}} \leq 1, \quad \forall k, \forall n, \forall \xi \\
& x_{k n \xi}^{\mathrm{EV}}=\sum_{c \in C} x_{k n c \xi}^{\mathrm{EV}, \mathrm{C}}, \quad \forall k, \forall n, \forall \xi
\end{aligned}
$$

- Day-ahead market constraints

$$
\begin{aligned}
& \left\{\leq p_{i e t \xi}^{\mathrm{D}} \leq p_{i}^{\mathrm{G}, \mathrm{C}}, \quad \forall i \in I^{\mathrm{G}, \mathrm{D}}, \forall t\right. \\
& 0 \leq p_{i e t \xi}^{\mathrm{D}} \leq p_{i}^{\mathrm{SP}, \mathrm{C}}, \quad \forall i \in I^{\mathrm{S}}, \forall t \\
& 0 \leq p_{i e t \xi}^{\mathrm{C}} \leq p_{i}^{\mathrm{SP}, \mathrm{C}}, \quad \forall i \in I^{\mathrm{S}}, \forall t \\
& p_{i e t \xi}^{\mathrm{D}}+s_{i e t \xi}^{\mathrm{D}}=U_{i e t}^{\mathrm{D}} p_{i}^{\mathrm{G}, \mathrm{C}}, \quad \forall i \in I^{\mathrm{G}, \mathrm{I}}, \forall t \\
& p_{i e t \xi}^{\mathrm{D}} \geq 0, \quad \forall i \in I^{\mathrm{G}, \mathrm{I}}, \forall t \\
& s_{i e t \xi}^{\mathrm{D}} \geq 0, \quad \forall i \in I^{\mathrm{G}, \mathrm{I}}, \forall t \\
& p_{\ell e t \xi}^{\mathrm{L}, \mathrm{D}}=\frac{1}{X_{\ell}}\left(\theta_{O(\ell) e t \xi}^{\mathrm{D}}-\theta_{F(\ell) e t \xi}^{\mathrm{D}}\right), \quad \forall \ell, \forall t
\end{aligned}
$$




$$
\begin{aligned}
& -P_{\max , \ell}^{\mathrm{L}} \leq p_{\ell e t \xi}^{\mathrm{L}, \mathrm{D}} \leq P_{\max , \ell}^{\mathrm{L}}, \quad \forall \ell, \forall t \\
& -\pi / 2 \leq \theta_{n e t \xi}^{\mathrm{D}} \leq \pi / 2, \quad \forall n, \forall t \\
& \sum_{i \in I_{n}} p_{i e t \xi}^{\mathrm{D}}-\sum_{i \in I_{n}^{\mathrm{S}}} p_{i e t \xi}^{\mathrm{C}}+\sum_{k \in K_{t}}\left(p_{k n e t \xi}^{\mathrm{EV}, \mathrm{DD}}-p_{k n e t \xi}^{\mathrm{EV}, \mathrm{DC}}\right)- \\
& \sum_{\ell \in L_{n}^{\mathrm{O}}} p_{\ell e t \xi}^{\mathrm{L}, \mathrm{D}}+\sum_{\ell \in L_{n}^{\mathrm{F}}} p_{\ell e t \xi}^{\mathrm{L}, \mathrm{D}}=L_{n e t \xi}^{\mathrm{D}}+\sum_{k \in K_{t}}\left(1-x_{k n \xi}^{\mathrm{EV}}\right) L_{k n e t \xi}^{\mathrm{EV}}, \\
& \forall n, \forall t \\
& 0 \leq c_{i e t \xi}^{\mathrm{U}} \leq p_{i}^{\mathrm{G}, \mathrm{C}}, \quad \forall i \in I^{\mathrm{G}, \mathrm{C}}, \forall t \\
& 0 \leq c_{i e t \xi}^{\mathrm{D}} \leq p_{i}^{\mathrm{G}, \mathrm{C}}, \quad \forall i \in I^{\mathrm{G}, \mathrm{C}}, \forall t \\
& 0 \leq c_{i e t \xi}^{\mathrm{U}} \leq p_{i}^{\mathrm{SP}, \mathrm{C}}, \quad \forall i \in I^{\mathrm{S}}, \forall t \\
& 0 \leq c_{i e t \xi}^{\mathrm{D}} \leq p_{i}^{\mathrm{SP}, \mathrm{C}}, \quad \forall i \in I^{\mathrm{S}}, \forall t
\end{aligned}
$$

- Real-time market constraints

$$
\begin{aligned}
& \left\{p_{i e t \xi \omega}^{\mathrm{R}}=p_{i e t \xi}^{\mathrm{D}}+r_{i e t \xi \omega}^{\mathrm{U}}-r_{i e t \xi \omega}^{\mathrm{D}}, \quad \forall i \in I^{\mathrm{G}, \mathrm{D}}, \forall t\right. \\
& 0 \leq p_{i e t \xi \omega}^{\mathrm{R}} \leq p_{i}^{\mathrm{G}, \mathrm{C}}, \quad \forall i \in I^{\mathrm{G}, \mathrm{D}}, \forall t \\
& p_{i e t \xi \omega}^{\mathrm{R}}-p_{i e t-1, \xi \omega}^{\mathrm{R}} \leq P_{\mathrm{up}, i}^{\mathrm{G}}, \quad \forall i \in I^{\mathrm{G}, \mathrm{D}}, \forall t \\
& p_{i e t-1, \xi \omega}^{\mathrm{R}}-p_{i e t \xi \omega}^{\mathrm{R}} \leq P_{\mathrm{dn}, i}^{\mathrm{G}}, \quad \forall i \in I^{\mathrm{G}, \mathrm{D}}, \forall t \\
& p_{i e t \xi \omega}^{\mathrm{R}}+s_{i e t \xi \omega}^{\mathrm{R}}=U_{i e t \xi \omega}^{\mathrm{R}} p_{i}^{\mathrm{G}, \mathrm{C}}, \quad \forall i \in I^{\mathrm{G}, \mathrm{I}}, \forall t \\
& s_{i e t \xi \omega}^{\mathrm{R}} \geq 0, \quad \forall i \in I^{\mathrm{G}, \mathrm{I}}, \forall t \\
& 0 \leq r_{i e t \xi \omega}^{\mathrm{U}} \leq c_{i e t \xi}^{\mathrm{U}}, \quad \forall i \in I^{\mathrm{G}, \mathrm{D}}, \forall t \\
& 0 \leq r_{i e t \xi \omega}^{\mathrm{D}} \leq c_{i e t \xi}^{\mathrm{D}}, \quad \forall i \in I^{\mathrm{G}, \mathrm{D}}, \forall t \\
& p_{\ell e t \xi \omega}^{\mathrm{L}, \mathrm{R}}=\frac{1}{X_{\ell}}\left(\theta_{O(\ell) e t \xi \omega}^{\mathrm{R}}-\theta_{F(\ell) e t \xi \omega}^{\mathrm{R}}\right), \forall \ell, \forall t \\
& -P_{\max , \ell}^{\mathrm{L}} \leq p_{\ell e t \xi \omega}^{\mathrm{L}, \mathrm{R}} \leq P_{\max , \ell}^{\mathrm{L}}, \quad \forall \ell, \forall t \\
& -\pi / 2 \leq \theta_{n e t \xi \omega}^{\mathrm{R}} \leq \pi / 2, \quad \forall n, \forall t
\end{aligned}
$$




$$
\begin{aligned}
& \sum_{i \in I_{n}}\left(r_{i e t \xi \omega}^{\mathrm{U}}-r_{i e t \xi \omega}^{\mathrm{D}}\right)+\sum_{i \in I_{n}^{\mathrm{R}}}\left(p_{i e t \xi \omega}^{\mathrm{R}}-p_{i e t \xi}^{\mathrm{D}}\right)- \\
& \sum_{k \in K_{n}}\left(r_{k n e t \xi \omega}^{\mathrm{EV}, \mathrm{U}}-r_{k n e t \xi \omega}^{\mathrm{EV}, \mathrm{D}}\right)+ \\
& \sum_{\ell \in L_{n}^{\mathrm{O}}}\left(p_{\ell e t \xi \omega}^{\mathrm{L}, \mathrm{R}}-p_{\ell e t \xi}^{\mathrm{L}, \mathrm{D}}\right)+\sum_{\ell \in L_{n}^{\mathrm{F}}}\left(p_{\ell t \xi \omega}^{\mathrm{L}, \mathrm{R}}-p_{\ell t \xi}^{\mathrm{L}, \mathrm{D}}\right)+ \\
& p_{n e t \xi \omega}^{\mathrm{UD}}=L_{n e t \xi \omega}^{\mathrm{R}}-L_{n e t \xi}^{\mathrm{D}}+ \\
& \sum_{k \in K_{t}}\left(1-x_{k n \xi}^{\mathrm{EV}}\right)\left(L_{k n e t \xi \omega}^{\mathrm{R}, \mathrm{EV}}-L_{k n e t \xi}^{\mathrm{D}, \mathrm{EV}}\right), \forall n, \forall t \\
& p_{i e t \xi \omega}^{\mathrm{SS}}=\gamma^{\mathrm{SS}, 0} p_{i}^{\mathrm{SE}, \mathrm{C}}, \quad \forall i \in I^{\mathrm{S}}, t=0 \\
& p_{i e t \xi \omega}^{\mathrm{SS}} \geq \gamma^{\mathrm{SS}, \mathrm{E}} p_{i}^{\mathrm{SE}, \mathrm{C}}, \quad \forall i \in I^{\mathrm{S}}, t=N_{\mathrm{T}} \\
& p_{i e t \xi \omega}^{\mathrm{SS}}=p_{i e t-1, \xi \omega}^{\mathrm{SS}}+\eta^{\mathrm{S}} p_{i e t \xi \omega}^{\mathrm{SC}}-\frac{1}{\eta^{\mathrm{S}}} p_{i e t \xi \omega}^{\mathrm{SD}}, \\
& \forall i \in I^{\mathrm{S}}, \forall t \\
& \gamma_{i}^{\mathrm{SSmin}} p_{i}^{\mathrm{SE}, \mathrm{C}} \leq p_{i e t \xi \omega}^{\mathrm{SS}} \leq p_{i}^{\mathrm{SE}, \mathrm{C}}, \quad \forall i \in I^{\mathrm{S}}, \forall t \\
& p_{i e t \xi \omega}^{\mathrm{SD}}=p_{i e t \xi}^{\mathrm{D}}-r_{i e t \xi \omega}^{\mathrm{UC}}+r_{i e t \xi \omega}^{\mathrm{DC}}, \forall i \in I^{\mathrm{S}}, \forall t \\
& p_{i e t \xi \omega}^{\mathrm{SC}}=p_{i e t \xi}^{\mathrm{C}}+r_{i e t \xi \omega}^{\mathrm{UD}}-r_{i e t \xi \omega}^{\mathrm{DD}}, \forall i \in I^{\mathrm{S}}, \forall t \\
& 0 \leq p_{i e t \xi \omega}^{\mathrm{SC}} \leq p_{i}^{\mathrm{SP}, \mathrm{C}}, \quad \forall i \in I^{\mathrm{S}}, \forall t \\
& 0 \leq p_{i e t \xi \omega}^{\mathrm{SD}} \leq p_{i}^{\mathrm{SP}, \mathrm{C}}, \quad \forall i \in I^{\mathrm{S}}, \forall t \\
& r_{i e t \xi \omega}^{\mathrm{U}}=r_{i e t \xi \omega}^{\mathrm{UC}}+r_{i e t \xi \omega}^{\mathrm{UD}}, \quad \forall i \in I^{\mathrm{S}}, \forall t \\
& 0 \leq r_{i e t \xi \omega}^{\mathrm{UC}} \leq p_{i e t \xi}^{\mathrm{C}}, \quad \forall i \in I^{\mathrm{S}}, \forall t \\
& 0 \leq r_{i e t \xi \omega}^{\mathrm{UD}} \leq p_{i}^{\mathrm{SP}, \mathrm{C}}-p_{i e t \xi}^{\mathrm{D}}, \quad \forall i \in I^{\mathrm{S}}, \forall t \\
& r_{i e t \xi \omega}^{\mathrm{D}}=r_{i e t \xi \omega}^{\mathrm{DC}}+r_{i e t \xi \omega}^{\mathrm{DD}}, \quad \forall i \in I^{\mathrm{S}}, \forall t \\
& 0 \leq r_{i e t \xi \omega}^{\mathrm{DC}} \leq p_{i}^{\mathrm{SP}, \mathrm{C}}-p_{i e t \xi}^{\mathrm{C}}, \quad \forall i \in I^{\mathrm{S}}, \forall t \\
& 0 \leq r_{i e t \xi \omega}^{\mathrm{DD}} \leq p_{i e t \xi}^{\mathrm{D}}, \quad \forall i \in I^{\mathrm{S}}, \forall t
\end{aligned}
$$




$$
\begin{aligned}
& p_{k n e t \xi \omega}^{\mathrm{EV}, \mathrm{S}}=N_{k n}^{\mathrm{EV}} P_{k n e \xi \omega}^{\mathrm{BS}, 0} x_{k n \xi}^{\mathrm{EV}}, \forall k, \forall n, t=t_{k}^{\mathrm{I}}-1 \\
& p_{k n e t \xi \omega}^{\mathrm{EV}, \mathrm{S}} \geq N_{k n}^{\mathrm{EV}} P_{k n e \omega}^{\mathrm{EV}, \mathrm{F}} x_{k n \xi}^{\mathrm{EV}}, \forall k, \forall n, t=t_{k}^{\mathrm{F}}-1 \\
& p_{k n e t \xi \omega}^{\mathrm{EV}, \mathrm{S}}=p_{k n e t-1, \xi \omega}^{\mathrm{EV}, \mathrm{S}}+\eta^{\mathrm{EV}} p_{k n e t \xi \omega}^{\mathrm{EV}, \mathrm{C}}-\frac{1}{\eta^{\mathrm{EV}}} p_{k n e t \xi \omega}^{\mathrm{EV}, \mathrm{D}} \text {, } \\
& \forall k, \forall n, t \in T_{k} \\
& N_{k n}^{\mathrm{EV}} P_{\min , k}^{\mathrm{EV}, \mathrm{S}} x_{k n \xi}^{\mathrm{EV}} \leq p_{k n e t \xi \omega}^{\mathrm{EV}, \mathrm{S}} \leq N_{k n}^{\mathrm{EV}} P_{\max , k}^{\mathrm{EV}, \mathrm{S}} x_{k n \xi}^{\mathrm{EV}} \text {, } \\
& \forall k, \forall n, \forall t \in T_{k} \\
& p_{k n e t \xi \omega}^{\mathrm{EV}, \mathrm{C}}=p_{k n e t \xi}^{\mathrm{EV}, \mathrm{DC}}-r_{k n e t \xi \omega}^{\mathrm{EV}, \mathrm{UC}}+r_{k n e t \xi \omega}^{\mathrm{EV}, \mathrm{DC}}, \\
& \forall k, \forall n, \forall t \in T_{k} \\
& p_{k n e t \xi \omega}^{\mathrm{EV}, \mathrm{D}}=p_{k n e t \xi}^{\mathrm{EV}, \mathrm{DD}}+r_{k n e t \xi \omega}^{\mathrm{EV}, \mathrm{UD}}-r_{k n e t \xi \omega}^{\mathrm{EV}, \mathrm{DD}}, \\
& \forall k, \forall n, \forall t \in T_{k} \\
& 0 \leq p_{k n e t \xi \omega}^{\mathrm{EV}, \mathrm{C}} \leq N_{k n}^{\mathrm{EV}} P_{\max }^{\mathrm{BPT}} x_{k n \xi}^{\mathrm{EV}}, \forall k, \forall n, \forall t \in T_{k} \\
& 0 \leq p_{k n e t \xi \omega}^{\mathrm{EV}, \mathrm{D}} \leq N_{k n}^{\mathrm{EV}} P_{\max }^{\mathrm{BPT}} x_{k n 2 \xi}^{\mathrm{EV}, \mathrm{C}}, \forall k, \forall n, \forall t \in T_{k} \\
& r_{k n e t \xi \omega}^{\mathrm{EV}, \mathrm{U}}=r_{k n e t \xi \omega}^{\mathrm{EV}, \mathrm{UC}}+r_{k n e t \xi \omega}^{\mathrm{EV}, \mathrm{UD}}, \forall k, \forall n, \forall t \in T_{k} \\
& 0 \leq r_{k n e t \xi \omega}^{\mathrm{EV}, \mathrm{UC}} \leq p_{k n e t \xi}^{\mathrm{EV}, \mathrm{DC}}, \forall k, \forall n, \forall t \in T_{k} \\
& 0 \leq r_{k n e t \xi \omega}^{\mathrm{EV}, \mathrm{UD}} \leq N_{k n}^{\mathrm{EV}} P_{\max }^{\mathrm{BPT}} x_{k n \xi}^{\mathrm{EV}}-p_{k n e t \xi}^{\mathrm{EV}, \mathrm{DD}}, \\
& \forall k, \forall n, \forall t \in T_{k} \\
& r_{k n e t \xi \omega}^{\mathrm{EV}, \mathrm{D}}=r_{k n e t \xi \omega}^{\mathrm{EV}, \mathrm{DC}}+r_{k n e t \xi \omega}^{\mathrm{EV}, \mathrm{DD}}, \forall k, \forall n, \forall t \in T_{k} \\
& 0 \leq r_{k n e t \xi \omega}^{\mathrm{EV}, \mathrm{DC}} \leq N_{k n}^{\mathrm{EV}} P_{\max }^{\mathrm{BPT}} x_{k n 2 \xi}^{\mathrm{EV}, \mathrm{C}}-p_{k n e t \xi}^{\mathrm{EV}, \mathrm{DC}}, \\
& \forall k, \forall n, \forall t \in T_{k} \\
& 0 \leq r_{k n e t \xi \omega}^{\mathrm{EV}, \mathrm{DD}} \leq p_{k n e t \xi}^{\mathrm{EV}, \mathrm{DD}}, \forall k, \forall n, \forall t \in T_{k} \\
& \}, \forall \omega \in \Omega\}, \forall e \in E, \forall \xi \in \Xi
\end{aligned}
$$

where $\Theta$ is the set of all optimization variables in problem (1)-(65), hereinafter denoted as problem (P1).

The objective function (1) to be minimized comprises: i) the expected investment cost of building new generating and storage units, ii) the expected cost of 
the economic incentives given to PEV users, iii) the expected cost of the energy and reserves scheduled in the day-ahead market, and iv) the expected cost of the deployed reserves and the unserved demand in the real-time market.

The first set of constraints of problem (P1) is related to the investment decisions. Constraints (2)-(4) limit the capacity to be built per technology. Constraints (5) define the rate between energy and power capacities in storage units.

The second set of constraints formulates the incentives offered to PEV users. This incentive is mathematically characterized by the variable $\lambda_{k n c}^{\mathrm{EV}}$ and represents the amount annually received by each PEV user belonging to group $k$, bus $n$ and control mode $c$. In particular, constraints (6)-(11) mathematically describe the stepwise price-quota curves used to compute the number of PEVs willing to leave the control of the charge to the SO according to the offered economic incentive. Figure 2 depicts an example of a 4-block stepwise price-quota curve. Observe that the percentage of controlled vehicles depicted in this curve $\left(\hat{X}_{k c m \xi}^{\mathrm{EV}}\right)$ grows as the offered incentive price increases $\left(\hat{\lambda}_{k c m \xi}^{\mathrm{EV}}\right)$. Binary variables $v_{k n c m}^{\mathrm{EV}}$ are equal to 1 if the offered price belongs to block $m$ for a given PEV group $k$, bus $n$, control mode $c$, and long-term scenario $\xi$, being 0 otherwise. In this paper, two different control modes are considered. The control mode denoted by $c=1$ represents charging control, whereas $c=2$ characterizes charging and discharging control, assuming vehicle-to-grid capability of PEVs. Note that the methodology behind the estimation of these curves is a complex econometric problem beyond the scope of this paper. For the interested reader, [21] proposes a practical procedure based on the Hotelling model to derive price-quota curves in the case of an electricity supplier of PEVs. 


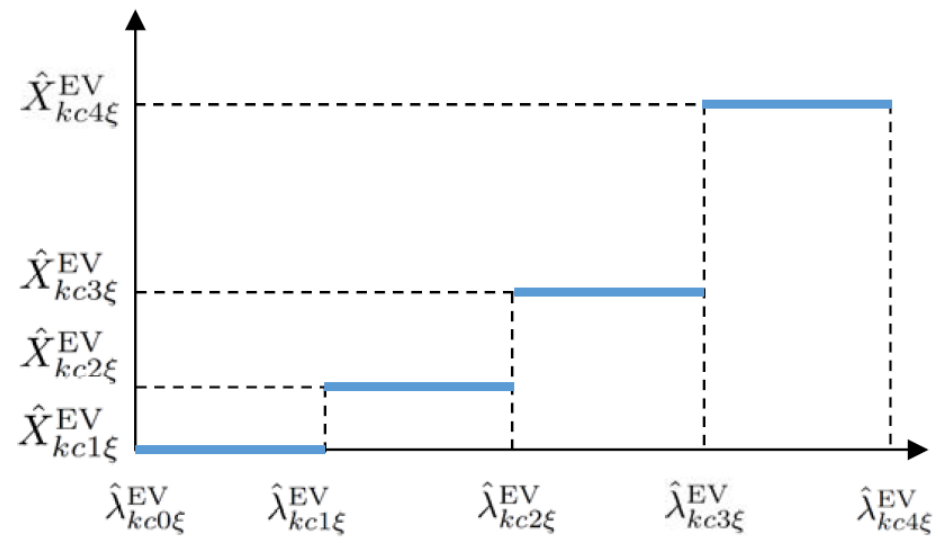

Figure 2: Price-quota curve

The third set of constraints describes the power system operation. The clearing of the day-ahead energy and reserve capacity market is formulated through constraints (12)-(25), while the real-time operation is defined by constraints (26)(65). Note that day-ahead market constraints must hold for each long-term scenario and each characteristic day, whereas real-time market constraints must also be satisfied in each short-term scenario. Constraints (12)-(17) limit the power scheduled by generating and storage units in the day-ahead market by the installed capacity. Constraints (18)-(20) define the transmission capacity limits in the day-ahead market. As usual in generation expansion models considering transmission constraints, (e.g. [2], [3], [7]-[11]), a DC linear representation of the network is used. Constraints (21) enforce the power balance in the day-ahead market. Constraints (22)-(25) limit the up and down reserve capacities scheduled by the dispatchable generating and storage units in the day-ahead market.

The technical constraints of the generating units in the real-time operation are formulated by constraints (26)-(31). Constraint (26) defines the power generated by dispatchable units considering the deployed up and down reserve, while constraints (27)-(29) impose the power output and the ramping limits, respectively. Constraints (30) and (31) limit the power output of the intermittent generating units considering their capacity and the power availability factor in the real-time operation. Constraints (34)-(36) impose the transmission capacity limits dur- 
ing the real-time operation. Constraint (37) enforces the power balance in the real-time operation considering the up and down deployed reserves, the difference between the available renewable power in the real-time and the day-ahead markets, the reserves deployed by the active groups of PEVs, the consequent difference in the power flow through the transmission lines, and the difference between the actual demand of consumers and non-controllable PEVs and the demand predicted in the day-ahead market. Constraint (38) establishes the initial energy level at each storage unit, whereas constraint (39) enforces the minimum energy level that must remain at the end of the day. Constraint (40) imposes the energy balance and constraints (41) establish the minimum and maximum energy limits in the storage. Constraints (42) and (45) are used to compute and limit the power charged and discharged in the real-time operation. According to constraints (46)-(48), the deployed up reserve by storage units may result from a reduction in the scheduled charged power (constraint (47)) and/or an increment in the discharged power respect to that scheduled in the day-ahead market (constraint (48)). In the same way, constraints (49)-(51) determine the deployed down reserve.

Finally, constraints (52)-(65) describe the state-of-charge of PEVs controlled by the SO. Constraints (52)-(55) are used to compute the energy stored in the batteries of PEVs in each period and scenario. Constraints (56)-(59) formulate the power charged and discharged by controlled PEVs of group $k$ at bus $n$. Similarly to constraints (46)-(51), constraints (60)-(65) are used to formulate the up and down reserves deployed by PEVs.

\section{Solution procedure}

Problem (P1) results in a large-scale mixed-integer linear programming problem. Since this problem may become intractable if a large number of scenarios is considered, we apply a procedure based on the Benders' decomposition technique to solve (P1) in reasonable computational times. Figure 3 represents graphically 
the proposed solution algorithm. Observe that if first-stage decisions are fixed, problem (P1) can be decomposed by long-term scenarios and characteristic days. Each of these resulting problems constitutes a subproblem in the Benders' decomposition algorithm that emulates the day-ahead market operation for a given long-term scenario and characteristic day. The investment and PEV incentive decisions are obtained by solving a master problem which constitutes a relaxation of (P1), where Benders' cuts obtained from solving subproblems are added in an iterative manner.

The practical implementation of Benders' decomposition to solve problem (P1) is explained in the rest of this section. In the following, superscript $(\nu)$ is used to denote the iteration counter. The interested reader is referred to [22] for further information on the practical implementation of Benders' decomposition.

\subsection{Subproblems}

As stated above, each subproblem emulates the day-ahead market operation for a given long-term scenario and characteristic day assuming that first-stage variables $p_{i}^{\mathrm{G}, \mathrm{C}}, p_{i}^{\mathrm{SE}, \mathrm{C}}, p_{i}^{\mathrm{SP}, \mathrm{C}}$ and $\lambda_{k n c}^{\mathrm{EV}}$ are fixed (i.e. obtained from the previous execution of the master problem). Note that, if $\lambda_{k n c}^{\mathrm{EV}}$ is known, the value of $x_{k n c \xi}^{\mathrm{EV}, \mathrm{C}}$ can be straightforwardly obtained through constraints (6)-(9). The solution of the subproblems provides the sensitivities of the capacity expansion cost. Then, the following problem is solved for each long-term scenario $\xi$ and each characteristic day $e$ : 


$$
\begin{aligned}
& \text { Minimize }_{\Theta_{\xi e}^{\mathrm{SP}}} \\
& \sum_{\xi \in \Xi} \tau_{\xi}\left[\sum _ { e \in E } w _ { e } \sum _ { t \in T } \left(\sum_{i \in I^{\mathrm{G}, \mathrm{D} \cup I^{\mathrm{S}}}}\left(C_{i e t \xi}^{\mathrm{D}} p_{i e t \xi}^{\mathrm{D}}+C_{i e t \xi}^{\mathrm{CU}} c_{i e t \xi}^{\mathrm{U}}+C_{i e t \xi}^{\mathrm{CD}} c_{i e t \xi}^{\mathrm{D}}\right)\right.\right. \\
& +\sum_{n \in N} C^{\mathrm{UD}} p_{n e t \xi}^{\mathrm{UD}, \mathrm{D}}+\sum_{\omega \in \Omega} \pi_{e \omega}\left(\sum _ { i \in \{ I ^ { \mathrm { G } , \mathrm { D } \cup I ^ { \mathrm { S } } \} } } \left(C_{i e t \xi}^{\mathrm{DU}} r_{i e t \xi \omega}^{\mathrm{U}}\right.\right. \\
& \left.\left.\left.\left.-C_{i e t \xi}^{\mathrm{DD}} r_{i e t \xi \omega}^{\mathrm{D}}\right)+\sum_{n \in N} C^{\mathrm{UD}} p_{n e t \xi \omega}^{\mathrm{UD}}\right)\right)\right]
\end{aligned}
$$

Subject to:

$$
\begin{aligned}
& \text { Constraints }(11)-(65) \\
& p_{i}^{\mathrm{G}, \mathrm{C}}=p_{i}^{\mathrm{G}, \mathrm{C},(\nu)}:\left(\alpha_{i e \xi}^{\mathrm{G}, \mathrm{C},(\nu)}\right), \quad \forall i \in I^{\mathrm{G}} \\
& p_{i}^{\mathrm{SE}, \mathrm{C}}=p_{i}^{\mathrm{SE}, \mathrm{C},(\nu)}:\left(\alpha_{i e \xi}^{\mathrm{SE}, \mathrm{C},(\nu)}\right), \quad \forall i \in I^{\mathrm{S}} \\
& p_{i}^{\mathrm{SP}, \mathrm{C}}=p_{i}^{\mathrm{SP}, \mathrm{C},(\nu)}:\left(\alpha_{i e \xi}^{\mathrm{SP}, \mathrm{C},(\nu)}\right), \quad \forall i \in I^{\mathrm{S}} \\
& x_{k n c \xi}^{\mathrm{EV}, \mathrm{C}}=x_{k n c \xi}^{\mathrm{EV}, \mathrm{C},(\nu)}:\left(\alpha_{k n c e \xi}^{\mathrm{EV}, \mathrm{C},(\nu)}\right), \quad \forall k, \forall n, \forall \xi
\end{aligned}
$$

where $\Theta_{\xi e}^{\mathrm{SP}}$ is the set of optimization variables of the subproblem associated with long-term scenario $\xi$ and day $e$. The optimal objective function of the subproblem associated with long-term scenario $\xi$ and characteristic day $e$ in iteration $(\nu)$ is denoted by $S P_{\xi e}^{(\nu)}$, which represents the expected operation cost on long-term scenario $\xi$ and characteristic day $e$.

\subsection{Master problem}

The master problem is a relaxed approximation of the original capacity expansion problem that is solved to determine the capacity investments in generation and storage units and the financial incentives offered to PEV users. The formu- 
lation of the master problem is:

$$
\begin{aligned}
& \sum_{\xi \in \Xi} \tau_{\xi}\left[\sum_{i \in I^{\mathrm{G}}} C_{i \xi}^{\mathrm{G}, \mathrm{C}} p_{i}^{\mathrm{G}, \mathrm{C}}+\sum_{i \in I^{\mathrm{S}}}\left(C_{i \xi}^{\mathrm{SE}, \mathrm{C}} p_{i}^{\mathrm{SE}, \mathrm{C}}+C_{i \xi}^{\mathrm{SP}, \mathrm{C}} p_{i}^{\mathrm{SP}, \mathrm{C}}\right)\right. \\
& \left.+\sum_{k \in K} \sum_{n \in N} \sum_{c \in C} \sum_{m \in M} N_{k n \xi}^{\mathrm{EV}} \hat{X}_{k m \xi}^{\mathrm{EV}} \lambda_{k c n m}^{\mathrm{EV}, \mathrm{A}}\right]+\beta
\end{aligned}
$$

Subject to:

$$
\begin{aligned}
& \text { Constraints (2)-(10) } \\
& \beta \geq \sum_{\xi \in \Xi} \tau_{\xi} \sum_{e \in E} w_{e}\left[S P_{\xi e}^{(\nu)}+\sum_{i \in I^{\mathrm{G}}} \alpha_{i e \xi}^{\mathrm{G}, \mathrm{C},(\nu)}\left(p_{i}^{\mathrm{G}, \mathrm{C}}-p_{i}^{\mathrm{G}, \mathrm{C},(\nu)}\right)+\right. \\
& \sum_{i \in I^{\mathrm{S}}}\left(\alpha_{i e \xi}^{\mathrm{SE}, \mathrm{C},(\nu)}\left(p_{i}^{\mathrm{SE}, \mathrm{C}}-p_{i}^{\mathrm{SE}, \mathrm{C},(\nu)}\right)+\alpha_{i e \xi}^{\mathrm{SP}, \mathrm{C},(\nu)}\left(p_{i}^{\mathrm{SP}, \mathrm{C}}-p_{i}^{\mathrm{SP}, \mathrm{C},(\nu)}\right)\right)+ \\
& +\sum_{k \in K, n \in N, c \in C} \alpha_{k n c e \xi}^{\mathrm{EV}, \mathrm{C},(\nu)}\left(x_{k n c \xi}^{\mathrm{EV}, \mathrm{C}}-x_{k n c \xi}^{\mathrm{EV}, \mathrm{C},(\nu)}\right), \forall \nu
\end{aligned}
$$

where $\Theta^{\mathrm{MP}}$ is the set of optimization variables of the master problem.The Benders' cuts formulated by constraints (74) are used in (72) to approximate the objective function (1). After solving the master problem, the optimal values of variables $p_{i}^{\mathrm{G}, \mathrm{C}}, p_{i}^{\mathrm{SE}, \mathrm{C}}, p_{i}^{\mathrm{SP}, \mathrm{C}}, \lambda_{k c n m}^{\mathrm{EV}, \mathrm{A}}$ and $\beta$ obtained in iteration $(\nu)$ are stored in parameters $p_{i}^{\mathrm{G}, \mathrm{C},(\nu)}, p_{i}^{\mathrm{SE}, \mathrm{C},(\nu)}, p_{i}^{\mathrm{SP}, \mathrm{C},(\nu)}, \lambda_{k c n m}^{\mathrm{EV}, \mathrm{A},(\nu)}$ and $\beta^{(\nu)}$, respectively. Observe that an additional Benders' cut is added to the master problem in each iteration $(\nu)$. 


\subsection{Upper and lower bounds}

Considering that each subproblem is solved for fixed values of the investment variables, an upper bound of the original problem can be computed as follows:

$$
\begin{aligned}
& z_{\mathrm{up}}^{(\nu)}=\sum_{\xi \in \Xi} \tau_{\xi}\left[\sum_{i \in I^{\mathrm{G}}} C_{i \xi}^{\mathrm{G}, \mathrm{C}} p_{i}^{\mathrm{G}, \mathrm{C},(\nu)}+\sum_{i \in I^{\mathrm{S}}}\left(C_{i \xi}^{\mathrm{SE}, \mathrm{C}} p_{i}^{\mathrm{SE}, \mathrm{C},(\nu)}+C_{i \xi}^{\mathrm{SP}, \mathrm{C}} p_{i}^{\mathrm{SP}, \mathrm{C},(\nu)}\right)\right. \\
& \left.+\sum_{k \in K} \sum_{n \in N} \sum_{c \in C} \sum_{m \in M} N_{k n \xi}^{\mathrm{EV}} \hat{X}_{k m \xi}^{\mathrm{EV}} \lambda_{k c n m}^{\mathrm{EV}, \mathrm{A},(\nu)}+\sum_{e \in E} w_{e} S P_{\xi e}^{(\nu)}\right]
\end{aligned}
$$

Since the master problem is a relaxed version of the original problem, the optimal value of its objective function (72) at each iteration $(\nu)$ constitutes an lower bound of the original problem:

$$
\begin{aligned}
& z_{\mathrm{lo}}^{(\nu)}=\sum_{\xi \in \Xi} \tau_{\xi}\left[\sum_{i \in I^{\mathrm{G}}} C_{i \xi}^{\mathrm{G}, \mathrm{C}} p_{i}^{\mathrm{G}, \mathrm{C},(\nu)}+\sum_{i \in I^{\mathrm{S}}}\left(C_{i \xi}^{\mathrm{SE}, \mathrm{C}} p_{i}^{\mathrm{SE}, \mathrm{C},(\nu)}+C_{i \xi}^{\mathrm{SP}, \mathrm{C}} p_{i}^{\mathrm{SP}, \mathrm{C},(\nu)}\right)\right. \\
& \left.+\sum_{k \in K} \sum_{n \in N} \sum_{c \in C} \sum_{m \in M} N_{k n \xi}^{\mathrm{EV}} \hat{X}_{k m \xi}^{\mathrm{EV}} \lambda_{k c n m}^{\mathrm{EV}, \mathrm{A},(\nu)}\right]+\beta^{(\nu)}
\end{aligned}
$$

If the difference between the upper and lower bounds is smaller than a prespecified tolerance the algorithm concludes and the optimal decisions are $p_{i}^{\mathrm{G}, \mathrm{C},(\nu)}$, $p_{i}^{\mathrm{SE}, \mathrm{C},(\nu)}, p_{i}^{\mathrm{SP}, \mathrm{C},(\nu)}$ and $\lambda_{k n c}^{\mathrm{EV},(\nu)}$. Otherwise, the algorithm continues.

It should be noted that Benders' decomposition cannot guarantee optimallity when applied to non-convex problems. However, the objective function of the master problem, as a function of the complicating variables, convexifies if a sufficient number of subproblems is considered. This result has been explained in [23] and [24]. This convexification allows a successful implementation of Benders' decomposition in practical exercises. 


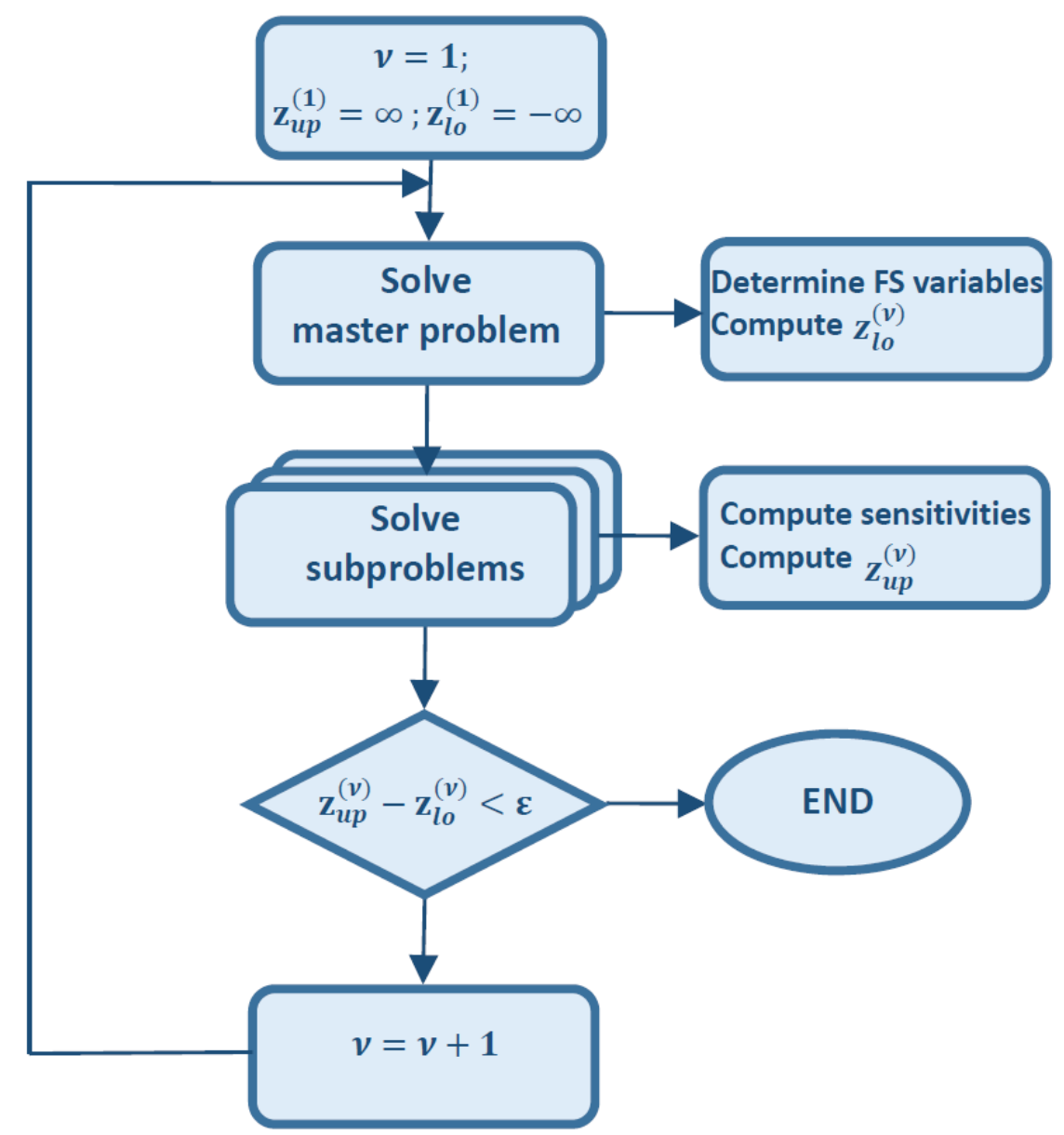

Figure 3: Flowchart representing the Benders' decomposition algorithm applied to solve problem (P1).

\section{Numerical results}

A realistic case study based on the isolated power system of LanzaroteFuerteventura (LZ-FV) in Spain has been used to analyze the performance of the proposed formulation. The objective of this case study is to determine the optimal generation capacity mix that should be installed at the end of year 2050 and the financial incentives offered to PEV users. It is assumed that all generating units that are currently at work will be decommissioned prior to the considered target year. For the sake of tractability, the target year has been represented by a set of 7 characteristic days that have been selected by using the scenario reduction algorithm described in [25]. 


\subsection{Input data}

The main input data describing the LZ-FV system is provided in [26] and [27]. This power system comprises 8 buses, 8 lines and a set of 45 candidate generating units to install. The technologies of these candidate units are diesel, gas, wind and solar PV. Due to limitation of space, we refer the interested reader to [27] for further information on the technical characteristics of the generating units, the transmission network, and consumption bids and generation offers in the day-ahead and real-time markets. Additional specific data follow.

\subsubsection{Candidate generating and storage units}

We assume that all thermal units that were at usage during year 2017 are candidate units to be installed again. The estimated potentials of renewable power plants are also considered to determine the maximum capacity of wind and PV power that can be installed in each bus of the system [28]. Table 1 provides the considered potentials of renewable units per bus and technology.

Table 1: Potentials of renewable capacity

\begin{tabular}{c|c|c|c|c|c}
\hline \hline \multirow{2}{*}{ Node } & \multicolumn{2}{|c|}{ Capacity (MW) } & \multirow{2}{*}{ Node } & \multicolumn{2}{c}{ Capacity (MW) } \\
\cline { 2 - 3 } \cline { 5 - 6 } & Wind & PV & & Wind & PV \\
\hline 1 & 61.0 & 64.2 & 5 & 39.0 & 16.8 \\
2 & 9.0 & 73.6 & 6 & 0.0 & 204.0 \\
3 & 0.0 & 13.8 & 7 & 0.0 & 78.1 \\
4 & 0.0 & 18.4 & 8 & 61.1 & 33.1 \\
\hline \hline
\end{tabular}

Additionally, 8 candidate storage facilities, one per bus, are considered. The maximum energy capacity that can be installed per bus is $100 \mathrm{MWh}$, and a typical relationship energy/power $\left(\gamma_{i}^{\mathrm{SP}}\right)$ equal to 6 has been considered.

\subsubsection{PEVs modeling}

Electric vehicles are grouped into three sets that have been defined based on the mobility study [29]. The description of the characteristics of each group is included in Table 2. Each electric vehicle is equipped with a $40 \mathrm{kWh}$ battery and it is considered that the average daily distance driven by each vehicle is $35 \mathrm{~km}$ 
[30]. The energy consumption is $0.196 \mathrm{kWh} / \mathrm{km}$. The efficiency of charging and discharging the batteries is equal to 0.88. Electric vehicles are charged under a peak power transfer rate of $11 \mathrm{~kW}$.

Table 2: PEV group characterization

\begin{tabular}{ccccc}
\hline \hline Type & $\begin{array}{c}\text { Beginning } \\
\text { hour }\end{array}$ & $\begin{array}{c}\text { Ending } \\
\text { hour }\end{array}$ & Duration & $\begin{array}{c}\text { Percentage } \\
\text { of PEVs }\end{array}$ \\
\hline 1 & 17.00 & 8.00 (next day) & 15 hours & $50 \%$ \\
2 & 20.00 & 8.00 (next day) & 12 hours & $35 \%$ \\
3 & 8.00 & 17.00 & 9 hours & $15 \%$ \\
\hline \hline
\end{tabular}

\subsubsection{Financial incentive}

The willingness of PEV users to leave the control of the charging processes of their vehicles to the SO is modeled with 10-block stepwise price-quota curves. These curves are generated for cases $c=\{1,2\}$ by discretizing two normal distributions with means 100 and $200 € / \mathrm{PEV} /$ year, respectively, and standard deviations equal to $25 € / \mathrm{PEV} /$ year. The resulting price-quota curves are depicted in Figure 4.

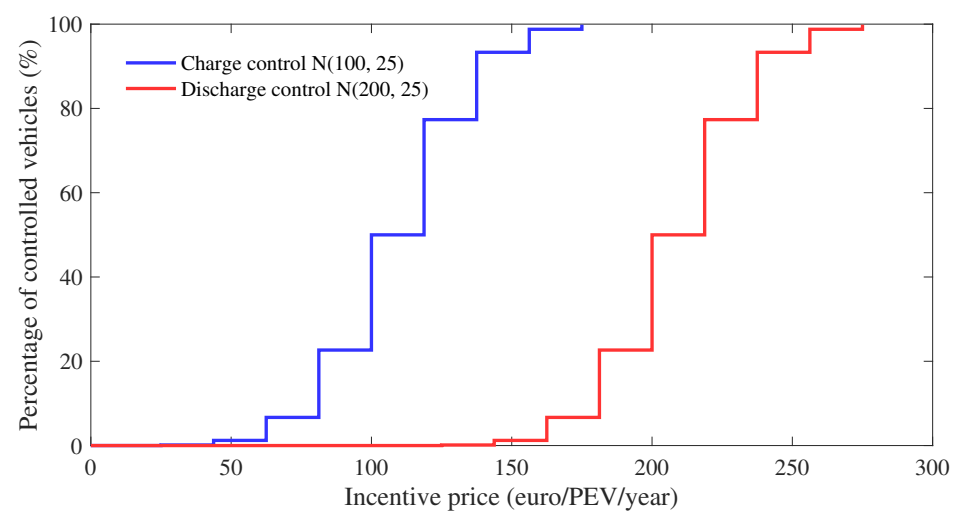

Figure 4: 10-block stepwise price-quota curve

\subsubsection{Long-term uncertainty}

The capital costs of renewable and storage units, the annual demand growth, the number of PEVs, and the controllability of PEVs are characterized as longterm uncertain parameters. For this purpose, we consider three values (low, 
medium and high) for each parameter according to the values provided in Table 3.

Table 3: Parameters modeling long-term scenarios

\begin{tabular}{lcccccc}
\hline \hline \multirow{2}{*}{ Parameters (\%) } & \multicolumn{2}{c}{ Low } & \multicolumn{2}{c}{ Medium } & \multicolumn{2}{c}{ High } \\
& Value & Prob. & Value & Prob. & Value & Prob. \\
\hline Var. of the cap. cost of renewables & -25.0 & 0.25 & 0.0 & 0.50 & 25.0 & 0.25 \\
Var. of the cap. cost of storages & -50.0 & 0.25 & 0.0 & 0.50 & 50.0 & 0.25 \\
Demand growth per year & 0.0 & 0.25 & 1.0 & 0.50 & 3.0 & 0.25 \\
Penetration of PEVs & 15.0 & 0.25 & 30.0 & 0.50 & 50.0 & 0.25 \\
Horizon. shift of price-quota curves & -25.0 & 0.25 & 0.0 & 0.50 & 25.0 & 0.25 \\
\hline \hline
\end{tabular}

The variation of the capital costs of renewable and storage units provided in Table 3 are computed over the values listed in Table 4, which includes the capital costs of the different generating technologies and storage units. These costs are afterwards annualized using a capital recovery factor with interest rate equal to $9 \%$ and a plant life time equal to 25 years. The demand growth included in Table 3 is applied to the actual demand in LZ-FV in year 2015. The penetration of PEVs is computed over the total number of vehicles in LZ-FV in 2015, which is equal to 101,648 vehicles. The uncertainty associated with the willingness of PEV users to delegate the control of the charge of their PEVs to the $\mathrm{SO}$ is modeled shifting horizontally the price-quota curves represented in Figure 4 using the values indicated in Table 3 .

Table 4: Capital costs

\begin{tabular}{c|c||c|c}
\hline \hline Technology & $\begin{array}{c}\text { Capital cost } \\
(€ / \mathrm{kW})\end{array}$ & Technology & $\begin{array}{c}\text { Capital cost } \\
(€ / \mathrm{kW})\end{array}$ \\
\hline Diesel & 900 & PV & 2000 \\
Gas & 750 & Storage (energy) & $40^{(*)}$ \\
Wind & 1400 & Storage (power) & 1000 \\
\hline \hline
\end{tabular}

Note that if three scenarios are defined for each of the five long-term uncertain parameters described above, a total number of $3^{5}=243$ scenarios are generated. For the sake of tractability, this set of scenarios has been reduced to 10 by using a scenario reduction technique, [25]. Note that this value allows to reach a compromise between accuracy and tractability and is consistent with the 
number of scenarios used in state-of-the-art capacity investment studies, [2]-[11]. The algorithm proposed in [25] is based on solving the full optimization problem as many times as long-term scenarios are initially generated (243) considering only one scenario each time. Afterwards, scenarios are selected according to cost associated with each single scenario. The performance of the scenario reduction algorithm is depicted in Figure 5. The cost associated with each single long-term scenario is represented in blue color, whereas red circles are used to denote the scenarios that are finally selected by the scenario reduction algorithm.

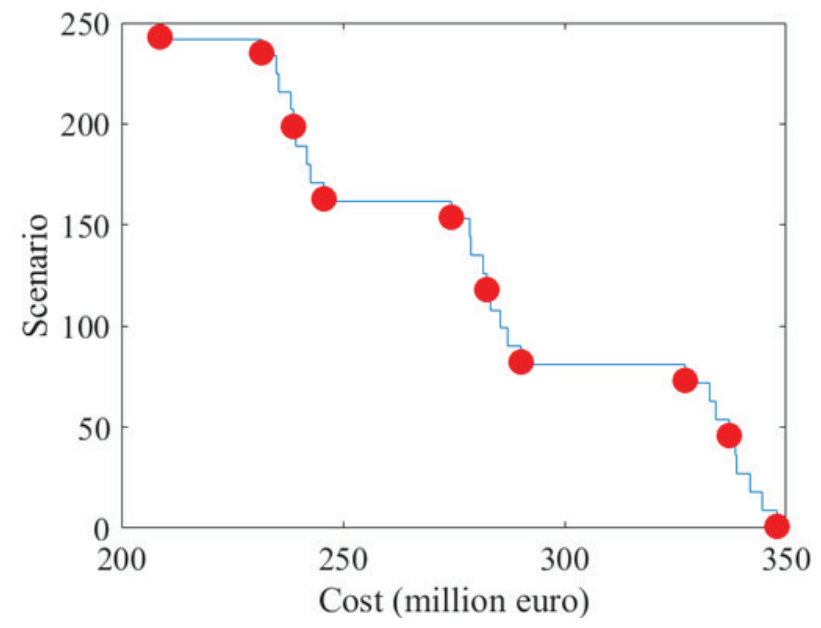

Figure 5: Long-term scenarios selection

\subsubsection{Short-term uncertainty}

The day-ahead demand, and the wind and solar PV availabilities are characterized as random variables and they are modeled using a set of scenarios. The ARIMA models proposed in [27] are used to characterize and randomly generate scenarios for demand, wind speed and solar radiation for each characteristic day. Considering this, an initial set of 100 scenarios is generated that has been afterwards reduced to 12 scenarios using the fast-forward selection algorithm described in $[31]$. 


\subsection{Results}

In this section, we provide the results obtained by using the input data described above. For the sake of comparison, three different cases are solved:

Case 1: In this case we consider that the $\mathrm{SO}$ is able to control the charge and discharge of those PEVs that wish to be controlled in exchange for a financial reimbursement. The installation of storage units is allowed.

Case 2: This case is similar to Case 1 but considering that PEVs cannot be controlled by the SO.

Case 3: This case is similar to Case 2 but considering that storages cannot be installed.

In particular, we show: i) the generating and storage capacity to be built in three different cases, which differ on whether or not the charging/discharging of the PEVs is controlled by the ISO, ii) the resulting number of PEVs under controlled in each node, iii) the day-ahead scheduling, and iv) the levelized cost of energy in the resulting power system.

All simulations are performed with CPLEX 12.6.1 using a server with four 3.0 GHz processors and 250 GB of RAM. The resulting problem has been solved using Benders' decomposition considering an optimality gap equal to $0.1 \%$. The number of constraints, continuous and binary variables in Case 1 are $12.3 \times 10^{6}$, $7.3 \times 10^{6}$, and 480 , respectively. All solution times are less than 43 hours. Note that this solution time is reasonable for a long-term decision-making problem. The economic and technical results are described in the following subsections.

\subsubsection{Expected cost}

Table 5 provides the resulting expected costs for each case. It is relevant to note that if the possibility of controlling the charge and discharge of PEVs is not considered (Case 2), the total expected cost increases $2.8 \%$ with respect to Case 1. In the same manner, if storages are not considered (Case 3), the expected cost increases $4.0 \%$ with respect to Case 1 . 
Table 5: Expected cost (million $€$ )

\begin{tabular}{cccccc}
\hline Case & $\begin{array}{c}\text { Annual inv. } \\
\text { gen. units }\end{array}$ & $\begin{array}{c}\text { Annual inv. } \\
\text { storage units }\end{array}$ & $\begin{array}{c}\text { Incentive } \\
\text { PEVs }\end{array}$ & Operation & Total \\
\hline Case 1 & 98.2 & 5.6 & 0.4 & 193.3 & 297.5 \\
Case 2 & 98.8 & 6.3 & 0.0 & 200.6 & 305.7 \\
Case 3 & 92.0 & 0.0 & 0.0 & 217.4 & 309.4 \\
\hline
\end{tabular}

\subsubsection{Computational performance}

Table 6 provides the computational size of the full problem formulated in Section 2.4, and of the master problem and subproblems resulting from applying Benders' decomposition in Case 1. The number of constraints in the master problem corresponds to the first iteration. The average solution times to solve the master problem and each of $10 \times 7=70$ subproblems in each iteration are 8 and 25 seconds, respectively. In total, 88 iterations are needed to reach an optimality gap equal to $0.1 \%$, which results in a total computational time of 43 hours.

Table 6: Size of the problems

\begin{tabular}{l|ccc}
\hline Problem & Full & Master & Subproblem \\
\hline Constraints (\#) & 12309 & 10763 & 177 \\
Continuous variables (\#) & 10763 & 1791 & 111 \\
Binary variables (\#) & 480 & 480 & 0 \\
\hline
\end{tabular}

\subsubsection{Installed capacity}

Table 7 provides the generation and storage capacity to be installed in each case for each technology. The percentage of the capacity installed over the potential of each technology is also included. Observe that the whole available capacity of wind power is installed in all cases. It is worth noting that if PEVs cannot be controlled by the SO (Case 2), the investments in storage capacity increase $12 \%$. If storages are not considered (Case 3), the installation of PV units decreases $20 \%$, whereas the installation of diesel and gas units increases $6 \%$ and $46 \%$, respectively. 
Table 7: Capacity to be installed (MW)

\begin{tabular}{l|ccccc}
\hline Case & Diesel & Gas & Wind & Solar PV & Storage \\
\hline \multirow{2}{*}{ Case 1 } & 272.3 & 39.6 & 170.0 & 222.7 & 37.2 \\
& $(78.4 \%)$ & $(7.4 \%)$ & $(100 \%)$ & $(45.4 \%)$ & $(27.9 \%)$ \\
\hline \multirow{2}{*}{ Case 2 } & 270.9 & 36.5 & 170.0 & 227.3 & 41.8 \\
& $(78.0 \%)$ & $(6.9 \%)$ & $(100 \%)$ & $(45.3 \%)$ & $(31.4 \%)$ \\
\hline \multirow{2}{*}{ Case 3 } & 289.6 & 57.9 & 170.0 & 178.2 & - \\
& $(83.4 \%)$ & $(10.9 \%)$ & $(100 \%)$ & $(35.5 \%)$ & $(-)$ \\
\hline
\end{tabular}

\subsubsection{Financial incentives for controllable PEVs}

Table 8 lists the resulting financial incentives offered by the SO to controllable PEVs in Case 1. Observe that the financial incentive offered to PEVs in groups 1 and 2 in charging control mode is higher than that offered to PEVs belonging to group 3. The reason for this result is that the SO may have a strong interest in placing the charge of PEVs during valley hours at night. However, this is not possible for group 3, which can only charge or discharge during the central part of the day, between 8:00 and 17:00.

Table 8: Financial incentives per PEV and per year for each PEV group (€/PEV/year)

\begin{tabular}{c|cc}
\hline PEV group & Charging & Charging/discharging \\
\hline 1 & 75 & 122 \\
2 & 75 & 122 \\
3 & 47 & 150 \\
\hline
\end{tabular}

\subsubsection{Levelized cost of energy}

In this subsection we analyze the resulting levelized cost of energy (LCOE) of each generating and storage technology. The LCOE is a measure of the cost of the energy generated by each technology, which is computed as the annualized investment cost divided by the annual energy production plus the incurred operating costs during the year. In the case of storage units, the operating costs are computed as the cost of the energy purchased in the day-ahead and real-time markets. Considering this, Tables 9 and 10 provide the expected number of fullload hours and LCOE per technology and case. It is observed that gas units are 
peak units that are at operation for a small number of hours during the year, yielding a high expected LCOE for this tehcnology. On the other hand, wind power units present the smallest LCOE among all considered technologies.

Table 9: Expected full-load hours per technology (hours)

\begin{tabular}{l|ccccc}
\hline Case & Diesel & Gas & Wind & Solar PV & Storage \\
\hline Case 1 & 4643 & 72 & 2217 & 1472 & 3495 \\
Case 2 & 4858 & 69 & 2218 & 1472 & 3433 \\
Case 3 & 4653 & 109 & 2187 & 1473 & - \\
\hline
\end{tabular}

Table 10: Expected LCOE per technology (€/MWh)

\begin{tabular}{l|ccccc}
\hline Case & Diesel & Gas & Wind & Solar PV & Storage \\
\hline Case 1 & 210.5 & $>10^{4}$ & 110.2 & 310.2 & 170.1 \\
Case 2 & 205.9 & $>10^{4}$ & 110.1 & 316.8 & 172.7 \\
Case 3 & 213.0 & $>10^{4}$ & 111.6 & 248.3 & - \\
\hline
\end{tabular}

\section{Summary and conclusions}

This paper presents a three-stage stochastic programming problem to analyze the generation capacity expansion of a power system considering storage units and PEVs. The willingness of PEVs to be controlled by the system operator is modeled by means of price-quota curves. Long- and short-term uncertainties are explicitly considered. The resulting problem is a large-scale mixed-integer linear programming problem that can be solved using decomposition techniques.

The numerical results obtained from a case study based on the LanzaroteFuerteventura power system indicate that investment decisions are affected by the controllability of the charge and discharge of PEVs. In particular, investments in storage facilities are significantly reduced if PEVs are controlled by the power system operator. Additionally, it has been observed that the annual operation cost of the system can be reduced $3.6 \%$ thanks to the controllability of PEVs. Besides, the installation of solar PVs reduces $20 \%$ if the flexibility provided by PEVs and storages is not considered. Another interesting result is that wind power units are massively installed in the analyzed power system independently of the presence of storage units and the controllability of PEVs. It should be also 
highlighted that the economical incentives offered to PEV users depend significantly of the interval of hours in which PEVs are available for being controlled. For instance, the incentives offered to PEVs whose charge can be controlled at night are $59.6 \%$ higher than those offered to vehicles not connected during the night. Conversely, those PEVs that are willing to discharge energy in the grid obtain an incentive $22.8 \%$ higher if they are connected during the central part of the day.

\section{Acknowledgment}

This work has been supported by the Ministry of Economy and Competitiveness of Spain under Project DPI2015-71280-R MINECO/FEDER, UE.

\section{References}

\section{References}

[1] Intergovernmental Panel on Climate Change (2018). Global warming of $1.5^{\circ} \mathrm{C}$. http://ipcc.ch/report/sr15/

[2] R. Domínguez, A. J. Conejo, M. Carrión. Toward fully renewable electric energy systems. IEEE Trans Power Syst, 30(1) (2015), pp. 316-326.

[3] S. Pineda, J. M. Morales, T. Boomsma. Impact of forecast errors on expansion planning of power systems with a renewables target. Euro Jour Oper Resear, 248 (2016), pp. 1113-1122.

[4] P. Krukanont, T. Tezuka. Implications of capacity expansion under uncertainty and value of information: The near-term energy planning of Japan. Energy, 32(10) (2007), pp. 1809-1824,

[5] Y. Liu, R. Sioshansi, A. J. Conejo. Multistage stochastic investment planning with multiscale representation of uncertainties and decisions. IEEE Trans on Power Syst, 33(1) (2018), pp. 781-791. 
[6] D. Min, J. Ryu, D. G. Choi. A long-term capacity expansion planning model for an electric power system integrating large-size renewable energy technologies. Computers and Operations Research, 96 (2018), pp. 244-255.

[7] X. Chen, J. Lv , M. B. McElroy, X. Han, C. P. Nielsen, J. Wen. Power system capacity expansion under higher penetration of renewables considering flexibility constraints and low carbon policies. IEEE Trans Power Syst, 33(6) (2018), pp. $6240-6253$.

[8] A. Sarid, M. Tzur. The multi-scale generation and transmission expansion model. Energy, 148(2018), pp. 977-991,

[9] S. Wogrin, D.F. Gayme. Optimizing storage siting, sizing, and technology portfolios in transmission-constrained networks. IEEE Trans on Power Syst, 30(6) (2014), pp. 3304-3313.

[10] B. Xu, Y. Wang, Y. Dvorkin, R. Fernandez-Blanco, C. Silva-Monroy, J.P. Watson, D.S. Kirschen. Scalable planning for energy storage in energy and reserve markets. IEEE Trans on Power Syst, 32(6) (2016), pp. 4515-4527.

[11] T. Qiu, B. Xu, Y. Wang, Y. Dvorkin, D.S. Kirschen. Stochastic multistage coplanning of transmission expansion and energy storage. IEEE Trans on Power Syst, 32(1) (2017), pp. 643-651.

[12] P. J. Ramírez, D. Papadaskalopoulos, G. Strbac. Co-optimization of generation expansion planning and electric vehicles flexibility. IEEE Trans Smart Grid, 7(3) (2016), pp. 1609-1619.

[13] P. Meneses de Quevedo, G. Muñoz-Delgado, J. Contreras. Impact of electric vehicles on the expansion planning of distribution systems considering renewable energy, storage and charging stations. IEEE Trans Smart Grid, 10(1) (2018), pp. $794-804$. 
[14] J. Aghaei, A. E. Nezhad, A. Rabiee, E. Rahimi. Contribution of Plug-in Hybrid Electric Vehicles in power system uncertainty management. Renewable and Sustainable Energy Reviews, 59 (2016), pp. 450 - 458.

[15] I. Momber, S. Wogrin, T. Gómez San Roman. Retail pricing: A bilevel program for PEV aggregator decisions using indirect load control. IEEE Trans Power Syst, 31(1) (2018), pp. 464--473.

[16] M. Alizadeh, H. T. Wai, A. Goldsmith, A. Scaglione. Retail and Wholesale Electricity Pricing Considering Electric Vehicle Mobility. IEEE Trans Control Netw Syst, 6(1) (2019), pp. 249 - 260.

[17] A. Ghosh, V. Aggarwal. Menu-Based Pricing for Charging of Electric Vehicles with Vehicle-to-Grid Service. IEEE Trans Veh Technol, 67(11) (2018), pp. $10268-10280$.

[18] A. Gomez-Exposito, A.J. Conejo, C. Canizares. Electric energy systems: analysis and operation. Boca Raton: CRC Press; 2008.

[19] K. Hoyland, S. W. Wallace. Generating Scenario Trees for Multistage Decision Problems. Manag Science, 47(2) (2001), pp. 295-307.

[20] A. J. Conejo, M. Carrión, J. M. Morales, Decision Making Under Uncertainty in Electricity Markets, Springer, New York, NY, 2010.

[21] M. Carrión. Determination of the selling price offered by electricity suppliers to electric vehicle users. To appear in IEEE Trans Smart Grids (2019).

[22] A. J. Conejo, E. Castillo, R. Mínguez, R. Garcia-Bertrand, Decomposition Techniques in Mathematical Programming, Springer-Verlag Berlin Heidelberg, 2006.

[23] D. P. Bertsekas, N. R. Sandell. Estimates of the duality gap for large-scale separable nonconvex optimization problems. In Proc. 21st IEEE Conf. Decision and Control, 21 (1982), pp. 782-785. 
[24] L. Baringo, A. J. Conejo. Wind Power Investment: A Benders Decomposition Approach. IEEE Trans on Power Syst, 27(1) (2012), pp. 433-441.

[25] J. M. Morales, S. Pineda, A. J. Conejo, M. Carrion. Scenario reduction for futures market trading in electricity markets. IEEE Trans Power Syst, 24(2) (2009), pp. 878-888.

[26] M. Asensio, J. Contreras. Stochastic Unit Commitment in Isolated Systems With Renewable Penetration Under CVaR Assessment. IEEE Trans Smart Grid, 7(3) (2016), pp. 1356-1367.

[27] M. Carrión, R. Domínguez, M. Cañas-Carretón, R. Zárate-Miñano. Scheduling isolated power systems considering electric vehicles and primary frequency response. Energy, 168 (2019), pp. 1192-1207.

[28] Government of the Canary Islands, Energetic plan of the Canary Islands, PECAN (2007). Available at: http://www.gobcan.es/ceic/energia/doc/planificacion/pecan/pecan2002.pdf

[29] Mobility report for residents in spain (MOVILIA), available at: http://www.fomento.es.

[30] M. Carrión, R. Zárate-Miñano. Operation of intermittent-dominated power systems with a significant penetration of plug-in electric vehicles. Energy, 90 (2015), pp. 827-835.

[31] Gröwe-Kuska N, Heitsch H, Römisch W. Scenario reduction and scenario tree construction for power management problems.Proc. IEEE Bologna Power Technology Conf. Bologna, Italy (2003). 